\title{
GAPS IN HOCHSCHILD COHOMOLOGY IMPLY SMOOTHNESS FOR COMMUTATIVE ALGEBRAS
}

\author{
Luchezar L. Avramov and Srikanth Iyengar
}

\begin{abstract}
The paper concerns Hochschild cohomology of a commutative algebra $S$, which is essentially of finite type over a commutative noetherian ring $K$ and projective as a $K$-module. For a finite $S$-module $M$ it is proved that vanishing of $\mathrm{HH}^{n}(S \mid K ; M)$ in sufficiently long intervals imply the smoothness of $S_{\mathfrak{q}}$ over $K$ for all prime ideals $\mathfrak{q}$ in the support of $M$. In particular, $S$ is smooth if $\operatorname{HH}^{n}(S \mid K ; S)=$ 0 for $(\operatorname{dim} S+2)$ consecutive $n \geq 0$.
\end{abstract}

\section{Introduction}

Let $K$ be a commutative noetherian ring, $S$ a commutative $K$-algebra, and $M$ an $S$-module. We let $\mathrm{HH}_{*}(S \mid K ; M)$ and $\mathrm{HH}^{*}(S \mid K ; M)$ denote, respectively, the Hochschild homology and the Hochschild cohomology of the $K$-algebra $S$ with coefficients in $M$. For each $n \in \mathbb{Z}$ there are canonical homomorphisms

$$
\begin{aligned}
& \lambda_{n}^{M}:\left(\wedge_{S}^{n} \Omega_{S \mid K}\right) \otimes_{S} M \longrightarrow \operatorname{HH}_{n}(S \mid K ; M) \\
& \lambda_{M}^{n}: \operatorname{HH}^{n}(S \mid K ; M) \longrightarrow \operatorname{Hom}_{S}\left(\wedge^{n} \Omega_{S \mid K}, M\right)
\end{aligned}
$$

of $S$-modules, where $\Omega_{S \mid K}$ is the $S$-module of $K$-linear Kähler differentials of $S$. Other concepts appearing the next result are defined following its statement.

Main Theorem. Let $K$ be a commutative noetherian ring and $S$ a commutative $K$-algebra essentially of finite type, flat as a $K$-module. For a prime ideal $\mathfrak{q}$ in $S$ and a finite $S$-module $M$ with $M_{\mathfrak{q}} \neq 0$ the following conditions are equivalent:

(i) The K-algebra $S_{\mathfrak{q}}$ is smooth.

(ii $\left.{ }_{*}\right)$ Each map $\left(\lambda_{n}^{M}\right)_{\mathfrak{q}}$ is bijective and the $S_{\mathfrak{q}}$-module $\Omega_{S_{\mathfrak{q}} \mid K}$ is projective.

(iii $\left.{ }_{*}\right)$ There exist non-negative integers $t, u$ of different parity satisfying

$$
\mathrm{HH}_{t}(S \mid K ; M)_{\mathfrak{q}}=0=\mathrm{HH}_{u}(S \mid K ; M)_{\mathfrak{q}}
$$

When the $K$-module $S$ is projective they are also equivalent to:

(ii*) Each map $\left(\lambda_{M}^{n}\right)_{\mathfrak{q}}$ is bijective.

(iii*) There exist non-negative integers t, $u$ of different parity satisfying

$$
\operatorname{HH}^{t+i}(S \mid K ; M)_{\mathfrak{q}}=0=\operatorname{HH}^{u+i}(S \mid K ; M)_{\mathfrak{q}} \quad \text { for } \quad 0 \leq i \leq \operatorname{dim}_{S_{\mathfrak{q}}} M_{\mathfrak{q}}
$$

Received by the editors November 15, 2004.

1991 Mathematics Subject Classification. Primary 13D03, 14B25. Secondary 14M10, $16 \mathrm{E} 40$.

Research partly supported by NSF grants DMS 0201904 (L.L.A) and DMS 0442242 (S.I.). 
Recall that one says that $S$ is essentially of finite type if it is a localization of a finitely generated $K$-algebra. A flat $K$-algebra $S$ essentially of finite type is smooth if the structure map $K \rightarrow S$ has geometrically regular fibers. Equivalently, for every homomorphism of rings $K \rightarrow \ell$, where $\ell$ is field, the ring $S \otimes_{K} \ell$ has finite global dimension. We say that an $S$-module $M$ is finite if it is finitely generated, and let $\operatorname{dim}_{S} M$ denote the Krull dimension of $M$.

The theorem incorporates several known results, discussed below. There are two new aspects to our characterizations of smoothness: the use of cohomology (with a couple of exceptions, earlier results used vanishing of homology) and the introduction of coefficients (all earlier results dealt with the case $M=S$ ). A special case of the theorem relates to a question of Happel [16, (1.4)]:

For a (not necessarily commutative) algebra $A$ over a field $K$, with $\operatorname{rank}_{K} A$ finite, does $\mathrm{HH}^{n}(A \mid K ; A)=0$ for $n \gg 0$ imply finite global dimension?

The next corollary provides a strong affirmative answer in the commutative case. This is in sharp contrast to the general situation, where the answer is negative: see the companion paper [11] by Buchweitz, Green, Madsen, and Solberg.

Corollary. Let $K$ be a field let $S$ be a commutative $K$-algebra, finite dimensional as a $K$-vector space. If $\mathrm{HH}^{n}(S \mid K ; S)=0$ for two non-negative values of $n$ of different parity, then $S$ is a product of separable field extensions of $K$.

Proof. The hypothesis $\operatorname{rank}_{K} S<\infty$ implies that $\operatorname{dim} S$ is 0 , and that $S$ is smooth precisely when it is a product of finite separable field extensions of $K$.

We place our result in the context of earlier work relating vanishing of Hochschild (co)homology and smoothness. As always, Spec $S$ denotes the set of prime ideal of $S$; its subset $\operatorname{Supp}_{S} M=\left\{\mathfrak{q} \in \operatorname{Spec} S \mid M_{\mathfrak{q}} \neq 0\right\}$ is the support of $M$.

Antecedents. Let $S$ be a $K$-algebra $S$ essentially of finite type, flat as a $K$ module. When citing results, a roman numeral in italic font font indicates the variant of the correspondingly numbered condition in the Main Theorem, where the hypothesis is assumed to hold for $M=S$ and for all $\mathfrak{q} \in \operatorname{Spec} S$.

The HKR Theorem. Hochschild, Kostant, and Rosenberg [18] (when $K$ is a perfect field) and André [1] (in general) proved (i) $\Longrightarrow\left(\right.$ ii $\left._{*}\right) \&$ (ii*). As $S$ is essentially of finite type, the $S$-module $\Omega_{S \mid K}$ is finite, so $\wedge_{S}^{n} \Omega_{S \mid K}=0$ holds for all $n \gg 0$, hence one always has $\left(\mathrm{ii}_{*}\right) \Longrightarrow\left(\mathrm{iii}_{*}\right)$ and $\left(\mathrm{ii}^{*}\right) \Longrightarrow$ (iii*).

Homological converses to the HKR Theorem. André [1] proved $\left(i i_{*}\right) \Longrightarrow(i)$.

$\left(i i i_{*}\right) \Longrightarrow(i)$ was proved by Avramov and Vigué-Poirrier [6] when $K$ is a field; by Campillo, Guccione, Guccione, Redondo, Solotar, and Villamayor [7] when, in addition, $\operatorname{char}(K)=0$; by Rodicio [22] in general.

Cohomological converses to the HKR Theorem. Assume $S$ is projective over $K$.

For a Gorenstein ring $S$ Blanco and Majadas [8] proved that $\mathrm{HH}^{n}(S \mid K ; S)=0$ for $(\operatorname{dim} S+2)$ consecutive values of $n \geq 0$ implies $S$ is smooth over $K$; this is subsumed in the implication (iii*) $\Longrightarrow$ (i) of the Main Theorem. In joint work 
with Rodicio [9] they showed that if $S$ is locally complete intersection over $K$, then $\mathrm{HH}^{2 n}(S \mid K ; S)=0$ or $\operatorname{Ker}\left(\lambda_{S}^{2 n}\right)=0$ for a single $n \geq 0$ implies $S$ is smooth.

Generalizations. The Main Theorem is a special case of a much more general result, Theorem (4.2), concerning gaps in $\operatorname{Tor}_{*}^{R}(S, M)$ and $\operatorname{Ext}_{R}^{*}(S, N)$ when $R$ is a noetherian ring, $S$ is an algebra retract of $R$, and $M$ is a complex of $S$-modules. For $\operatorname{Tor}_{*}^{R}(S, S)$ that result is due to Rodicio [22]. However, to prove (iii*) $\Longrightarrow$ (i) in the Main Theorem, even for $M=S$, we do need to use complexes.

Conventions. In the rest of this article all rings are assumed to be commutative. A local ring is a noetherian ring that has a unique maximal ideal. A local homomorphism is a homomorphism of rings, whose source and target are local and which maps maximal ideal into maximal ideal.

\section{Closed homomorphisms}

In this section $\varphi:(R, \mathfrak{m}, k) \rightarrow S$ is a surjective local homomorphism.

We recall a general construction due to Tate [24]. More details about Tate resolutions and acyclic closures can be found in the original paper, in the book of Gulliksen and Levin [14, Chapter I], or in the survey [2, Chapter 6].

1.1. Tate resolutions. For each positive integer $n$ let $X_{n}$ denote a free graded $R$-module concentrated in degree $n$; furthermore, $R\left\langle X_{n}\right\rangle$ denotes the exterior algebra on $X_{n}$ if $n$ is odd, and the divided powers algebra on $X_{n}$ if $n$ is even; in the latter case, $x^{(i)}$ denotes the $i$ th divided power of $x \in X_{n}$.

A Tate resolution of $\varphi$ is a DG (= differential graded) algebra $G$ having a system of divided powers compatible with the action of the differential and a filtration $\left\{G^{(p)}\right\}_{p \geq 0}$ by DG subalgebras with divided powers, such that

(0) $G^{(0)}=R$ and $G^{(p-1)} \subseteq G^{(p)}$, for $p \geq 1$.

(1) $G^{(p)}=G^{(p-1)} \otimes_{R} R\left\langle\bar{X}_{p}\right\rangle$ as graded $R$-modules, for $p \geq 1$.

(2) $\partial\left(x^{(i)}\right)=\partial(x) x^{(i-1)}$ for all $i \geq 1$ when $|x|$ is even and positive.

(3) $\mathrm{H}_{0}\left(G^{(p)}\right)=S$ for $p \geq 1$.

(4) $\mathrm{H}_{i}\left(G^{(p)}\right)=0$ for $1 \leq i<p$.

(5) $G=\bigcup_{p \geqslant 0} G^{(p)}$.

Forgetting the multiplicative structures, $G$ is a free resolution of $R$ over $S$. A Tate resolution always exists: form DG algebras satisfying conditions (0) through (4) by induction on $p$, then use $(5)$ to define $G$; see $[24, \S 2]$. Control may be exercised at each step of the process.

As starting point, one may choose any surjective $R$-linear map

$$
\delta_{1}: X_{1} \longrightarrow \operatorname{Ker}(\varphi)
$$

and define the differential on $G^{(1)}$ so that its restriction to $X_{1}$ is the composition of $\delta_{1}$ with the inclusion $\operatorname{Ker}(\varphi) \subseteq R=G_{0}^{(0)}$. If $\boldsymbol{e}_{1}$ is a basis for $X_{1}$, then $\delta_{1}\left(\boldsymbol{e}_{1}\right)$ generates the ideal $\operatorname{Ker}(\varphi)$ and $G^{(1)}$ is the Koszul complex on $\delta\left(\boldsymbol{e}_{1}\right)$.

For each $p \geq 2$ one may choose any surjective $R$-linear map

$$
\delta_{p}: X_{p} \rightarrow \mathrm{H}_{p-1}\left(G^{(p-1)}\right)
$$


lift it to a homomorphism $\widetilde{\delta}_{p}: X_{p} \rightarrow \mathrm{Z}_{p-1}\left(G^{(p-1)}\right)$, and define a differential on $G^{(p)}$, which on $X_{p}$ is the composition of $\widetilde{\delta}_{p}$ with $\mathrm{Z}_{p-1}\left(G^{(p-1)}\right) \subseteq G_{p-1}^{(p-1)}=G_{p-1}^{(p)}$.

1.2. Acyclic closures. An acyclic closure of $\varphi$ is a Tate resolution obtained by choosing for each $p \geq 1$ the map $\delta_{p}$ in (1.1) to be a projective cover.

Let $G$ be an acyclic closure of $\varphi$ and let $G^{\prime}$ be a Tate resolution of $\varphi$. There exists then a morphism $\gamma: G \rightarrow G^{\prime}$ of DG $R$-algebras with divided powers, and for any such morphism the homomorphism of $R$-modules $\gamma_{n}: G_{n} \rightarrow G_{n}^{\prime}$ is a split injection. If $G^{\prime}$ is also an acyclic closure of $\varphi$, then $\gamma$ is an isomorphism, and it induces an isomorphism $G^{(p)} \rightarrow G^{\prime(p)}$ for each $p \geq 0$.

In particular, the pth stage $G^{(p)}$ of an acyclic closure $G$ of $\varphi$ is independent, up to isomorphism, of the choice of $G$.

The next remark is immediate from the construction of acyclic closures.

1.2.1. $G^{(1)}$ is the Koszul complex $E$ on a minimal generating set for $\operatorname{Ker}(\varphi)$.

We introduce two numerical invariants of $\varphi$, for use throughout the paper. Letting $\nu_{S}(N)$ denote the minimal number of generators an $S$-module $N$, we set

$$
\varepsilon_{2}(\varphi)=\nu_{S}(\operatorname{Ker}(\varphi)) \quad \text { and } \quad \varepsilon_{3}(\varphi)=\nu_{S}\left(\mathrm{H}_{1}(E)\right)
$$

These are part of the deviations of $\varphi$; see [5, (2.5)]. The first assertion below is clear; the second one is a standard characterization of regular sequences.

1.2.2. $\varepsilon_{2}(\varphi)=0$ if and only if $\varphi=\mathrm{id}^{R}$.

1.2.3. $\varepsilon_{3}(\varphi)=0$ if and only if $\varphi$ is generated by a regular sequence.

A complex $F$ of finite free $R$-modules is said to be minimal if $\partial(F) \subseteq \mathfrak{m} F$. For each integer $p \geq 1$, the construction of the $p$ th stage $F_{\leqslant p}$ of a minimal resolution of $S$ adds to $F_{\leqslant p-1}$ a single new free module in degree $p$.

In contrast, the construction of the $p$ th stage $G^{(p)}$ of an acyclic closure of $\varphi$ adds to $G^{(p-1)}$ shifts of every free module present in it: finitely many shifts appear when $p$ is odd, and infinitely many when $p$ is even. Thus, when the resolution of $S$ over $R$ provided by an acyclic closure is minimal, one has a certain control of the growth of that resolution.

This explains our interest in the class of maps described below.

1.3. Closed homomorphisms. We say that the homomorphism $\varphi$ is closed if some acyclic closure $G$ of $\varphi$ is a minimal resolution of $S$ over $R$.

A celebrated result of Gulliksen [13] and Schoeller [23] can be read as follows:

1.3.1. The canonical surjection $R \rightarrow k$ is closed for every $R$.

To state an extension, we recall that the homomorphism $\varphi$ is large if the map

$$
\operatorname{Tor}_{n}^{\varphi}(k, k): \operatorname{Tor}_{n}^{R}(k, k) \rightarrow \operatorname{Tor}_{n}^{S}(k, k)
$$

is surjective for each $n$. The notion was introduced by Levin [20]. The following theorem of Avramov and Rahbar-Rochandel, see [20, (2.5)], provides a significant supply of closed homomorphisms. 
1.3.2. Every large homomorphism is closed.

The last result will be applied through the following observation:

1.3.3. If there is a homomorphism of rings $\psi: S \rightarrow R$ with $\varphi \circ \psi=\mathrm{id}^{S}$, then

$$
\operatorname{Tor}_{n}^{\varphi}(k, k) \circ \operatorname{Tor}_{n}^{\psi}(k, k)=\operatorname{Tor}_{n}^{\varphi \circ \psi}(k, k)=\operatorname{Tor}_{n}^{\mathrm{id}^{S}}(k, k)=\mathrm{id}^{\operatorname{Tor}_{n}^{S}(k, k)}
$$

by functoriality; thus, $\operatorname{Tor}_{n}^{\varphi}(k, k)$ is surjective, hence $\varphi$ is large, and so closed.

In this paper we are mostly interested in obtaining lower bounds on the sizes of the $S$-modules $\operatorname{Tor}_{n}^{R}(S, M)$ and $\operatorname{Ext}_{R}^{n}(S, M)$. For that purpose we use properties of $\varphi$ that are weaker than closure.

1.4. Partly closed homomorphisms. Let $G^{(p)}$ be as in (1.2) for some $p \geq 1$ and $F$ be a minimal free resolution of the $R$-module $S$. As $\mathrm{H}_{0}\left(G^{(p)}\right)=S$ and each $G_{n}^{(p)}$ is $R$-free, the augmentation $G^{(p)} \rightarrow S$ lifts to a comparison morphism

$$
\gamma^{(p)}: G^{(p)} \rightarrow F
$$

We say that $\varphi$ is $p$-closed if $\gamma_{n}^{(p)}$ has an $R$-linear left inverse for each $n \in \mathbb{Z}$.

A homomorphism $\gamma$ of free $R$-modules of finite rank has a left inverse if and only if the map $k \otimes_{R} \gamma$ is injective. This yields an alternative description:

1.4.1. The homomorphism $\varphi$ is $p$-closed if and only if $G^{(p)}$ is minimal and the induced map $\mathrm{H}\left(k \otimes_{R} \gamma^{(p)}\right): k \otimes_{R} G^{(p)} \rightarrow \operatorname{Tor}^{R}(k, S)$ is injective.

1.4.2. If the homomorphism $\varphi$ is p-closed, $G^{\prime}$ an acyclic closure of $\varphi, F^{\prime}$ is a resolution of $S$ by finite free $R$-modules, and $\gamma^{\prime(p)}: G^{\prime(p)} \rightarrow F^{\prime}$ is a comparison morphism, then $\gamma_{n}^{\prime(p)}$ has a left inverse for each $n \in \mathbb{Z}$.

Indeed, (1.2) yields an isomorphism $\alpha: G^{(p)} \rightarrow G^{\prime(p)}$ of DG algebras over $R$, so $G^{\prime(p)}$ is minimal by (1.4.1). For any comparison morphism $\beta: F^{\prime} \rightarrow F$, the morphisms $\gamma^{(p)}$ and $\beta \gamma^{\prime(p)} \alpha$ are homotopic. Thus, $\mathrm{H}\left(k \otimes_{R} \gamma^{(p)}\right)$ factors as

$$
k \otimes_{R} G^{(p)} \stackrel{k \otimes_{R} \alpha}{\longrightarrow} k \otimes_{R} G^{\prime(p)} \stackrel{\mathrm{H}\left(k \otimes_{R} \gamma^{\prime(p)}\right)}{\longrightarrow} \mathrm{H}\left(k \otimes_{R} F^{\prime}\right) \stackrel{\mathrm{H}\left(k \otimes_{R} \beta\right)}{\longrightarrow} k \otimes_{R} F
$$

It follows that $\mathrm{H}\left(k \otimes_{R} \gamma^{\prime(p)}\right)$ is injective, see (1.4.1), hence $\gamma_{n}^{\prime(p)}$ is split injective.

1.4.3. Let $R^{\prime}$ be a local ring, let $\rho: R \rightarrow R^{\prime}$ be a faithfully flat local homomorphism, set $S^{\prime}=R^{\prime} \otimes_{R} S$, and let $\rho^{\prime}: R^{\prime} \rightarrow S^{\prime}$ denote the induced homomorphism. The map $\varphi$ is $p$-closed if and only if so is $\varphi^{\prime}$.

Indeed, $R^{\prime} \otimes_{R} G$ is an acyclic closure of $\varphi^{\prime}$ if $G$ is one of $\varphi$; see [14, (1.9.8)].

Next we place the properties discussed above in a familiar context, focusing on the case $p \leq 2$ because these are the classes of maps important for this paper. 
1.5. Comparisons. The homomorphism $\varphi$ is said to be complete intersection (or c.i., for short) if $\operatorname{Ker}(\varphi)$ is generated by a regular sequence. Clearly, one has

$$
\text { c.i. } \Longrightarrow \text { closed } \Longrightarrow \text { 2-closed } \Longrightarrow \text { 1-closed }
$$

1.5.1. The first implication is obviously strict; see for instance (1.3.1).

1.5.2. The canonical map from $R=k[[x, y]] /\left(x^{2}, x y\right)$ to $S=R /\left(y^{2}\right)$ is 1-closed, but not 2-closed: apply (1.4.2) to the commutative diagram

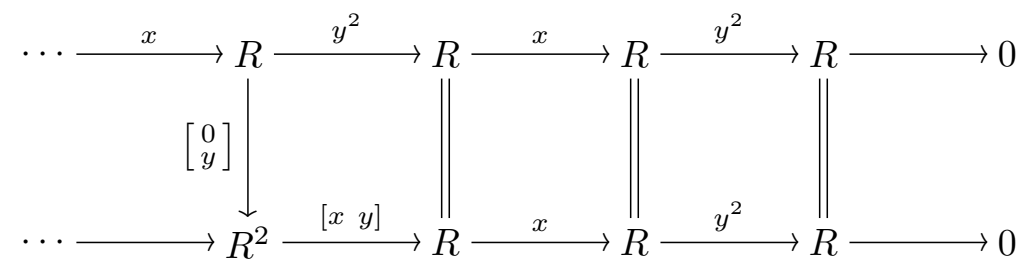

whose top row is the beginning of the second stage of an acyclic closure of $\varphi$ and whose bottom row is the beginning of a minimal resolution of $S$ over $R$.

1.5.3. We do not whether every 2-closed homomorphisms is actually closed.

Except for the name, 1-closed homomorphisms have appeared in literature.

1.6. One-closed homomorphisms. As defined, $p$-closure requires $\gamma_{n}^{(p)}$ to be split injective for each $n$. However, 1-closure can be detected in a single degree.

Lemma 1.6.1. Let $E$ be the Koszul complex on a minimal generating set for the ideal $\operatorname{Ker}(\varphi)$ and let $\gamma: E \rightarrow F$ be a comparison morphism to a minimal free resolution of $S$ over $R$. The following conditions are equivalent:

(1) The homomorphism $\varphi$ is 1-closed.

(2) For $c=\varepsilon_{2}(\varphi)$ the map $\left(k \otimes_{R} \gamma_{c}\right):\left(k \otimes_{R} E_{c}\right) \rightarrow \operatorname{Tor}_{c}^{R}(k, S)$ is injective.

Proof. Observation (1.2.1) and property (1.4.1) show that (1) implies (2).

For the converse, note that the isomorphism $k \otimes_{R} E \cong \wedge k^{c}$ of graded $k$ algebras shows that the socle of $k \otimes_{R} E$ is $k \otimes_{R} E_{c}$. As $k \otimes_{R} \gamma$ is a homomorphism of graded $k$-algebras, when $k \otimes_{R} \gamma_{c}$ is injective so is $k \otimes_{R} \gamma$; now use (1.4.2).

The preceding description brings to light a connection between 1-closure for parameter ideals and Hochster's Canonical Element Conjecture, see [19].

1.6.2. Let $R$ be a local ring. The following conditions are equivalent:

(1) The Canonical Element Conjecture holds for $R$.

(2) For each system of parameters $\boldsymbol{p}$ of $R$ the map $\varphi: R \rightarrow R /(\boldsymbol{p})$ is 1-closed.

Indeed, Roberts [21] has proved that the Canonical Element Conjecture holds for $R$ if and only if for each free resolution $F$ of $R /(\boldsymbol{p})$ over $R$ and each comparison morphism $\kappa: E \rightarrow F$, the induced map $\left(k \otimes_{R} E_{c}\right) \rightarrow \mathrm{H}_{c}\left(k \otimes_{R} F\right)$ is injective; another proof of his result is given by Huneke and Koh [17, (1.3)]. Thus, the desired equivalence is contained in (1.4.2) and Lemma (1.6.1). 
The hypothesis on $R$ in the next theorem reflects the use in its proof of a result of Bruns [10], which in turn relies on the Improved New Intersection Theorem.

Theorem 1.6.3. Let $\varphi: R \rightarrow S$ be a 1-closed homomorphism and assume $R$ contains a field as a subring. If $\operatorname{pd}_{R} S$ is finite, then $\varphi$ is complete intersection.

Proof. Set $I=\operatorname{Ker} \varphi$ and $c=\varepsilon_{2}(\varphi)$. The Koszul complex $E=G^{(1)}$ on a minimal generating set of $I$ yields an injection $\kappa: k \otimes_{R} E \rightarrow \operatorname{Tor}^{R}(k, S)$, see (1.4.1).

Since $\operatorname{pd}_{R} S$ is finite, [10, Lemma 2] yields $\kappa_{i}=0$ for $i>\operatorname{height} I$. By construction one has $\operatorname{rank}_{R} E_{1}=c$, and this implies $c \leq$ height $I$. The reverse inequality always holds, due to the Principal Ideal Theorem, hence one gets height $I=c$. On the other hand, $\operatorname{pd}_{R} S<\infty$ implies that height $I$ equals the maximal length of an $R$-regular sequence in $I$, see [3, (2.5)]. We concude that $I$ can be generated by an $R$-regular sequence, as desired.

\section{Bounds on homology}

The main result of this section is a condition for a 2-closed homomorphism to be c.i. When $\varphi$ admits a section and $M=S$ it specializes to a result of Rodicio, [22, Theorem 1]. The reason for dealing with complexes, rather than just with modules, will become apparent in the proof of Theorem (3.1).

Theorem 2.1. Let $\varphi: R \rightarrow S$ be a 2-closed local homomorphism and $M a$ complex of $S$-modules with $\mathrm{H}(M)$ degreewise finite and bounded below.

If there exist integers $t, u \geq \inf \mathrm{H}(M)$ of different parity, such that

$$
\operatorname{Tor}_{t}^{R}(S, M)=0=\operatorname{Tor}_{u}^{R}(S, M)
$$

then the homomorphism $\varphi$ is complete intersection.

We comment on notions and notation used in the theorem and its proof.

2.2. For definitions of Tor and Ext for complexes we refer to [25]. When their arguments are modules (modules are always identified with complexes concentrated in degree 0) these are the classical derived functors. We set

$$
\begin{aligned}
\inf \mathrm{H}(M) & =\inf \left\{n \mid \mathrm{H}_{n}(M) \neq 0\right\} \\
\sup \mathrm{H}(M) & =\sup \left\{n \mid \mathrm{H}_{n}(M) \neq 0\right\}
\end{aligned}
$$

When $\inf \mathrm{H}(M)$ (respectively, $\sup \mathrm{H}(M)$ ) is finite we say that $\mathrm{H}(M)$ is bounded below (respectively, above). If $H(M)$ is bounded on either side, then $\mathrm{H}(M) \neq 0$, because $\mathrm{H}(M)=0$ is equivalent to $\inf \mathrm{H}(M)=\infty$, and also to $\sup \mathrm{H}(M)=-\infty$.

For each integer $j$ a complex $\Sigma^{j} M$ is defined by

$$
\Sigma^{j}(M)_{n}=M_{n-j} \quad \text { and } \quad \partial_{n}^{\Sigma^{j} M}=(-1)^{|j|} \partial_{n-j}^{M}
$$

Morphisms of complexes are chain maps of degree 0. A quasiisomorphism is a morphism that induces isomorphisms in homology in all degrees; we tag quasiisomorphisms with the symbol $\simeq$, and isomorphisms with $\cong$.

We deduce Theorem (2.1) from the following, much stronger, result. 
Theorem 2.3. Let $\varphi: R \rightarrow S$ be a 2-closed local homomorphism, set $c=\varepsilon_{2}(\varphi)$ and $d=\varepsilon_{3}(\varphi)$. If $M$ is a complex of $S$-modules with $\mathrm{H}(M)$ degreewise finite and bounded below, then for $i=\inf \mathrm{H}(M)$ and $m=\nu_{S}\left(\mathrm{H}_{i}(M)\right)$ one has inequalities

$$
\begin{gathered}
\nu_{S}\left(\operatorname{Tor}_{n+i}^{R}(S, M)\right) \geq m \cdot\left(\begin{array}{l}
c \\
n
\end{array}\right) \quad \text { for } \quad 0 \leq n \leq c \\
\nu_{S}\left(\operatorname{Tor}_{2 n+i+c}^{R}(S, M)\right) \geq m \cdot\left(\begin{array}{c}
n+d-1 \\
d-1
\end{array}\right) \quad \text { for } \quad 1 \leq n
\end{gathered}
$$

The proof uses a general lemma in homological algebra, presented below.

2.4. Let $T$ be a covariant additive functor from the category of complexes of $S$ modules to the category of graded $S$-modules; for each complex $M$ of $S$-modules we write $T_{n}(M)$ for the component in degree $n$ of the graded $S$-module $T(M)$. Assume, furthermore, that $T$ has the following properties:

(a) $T$ preserves quasiisomorphisms.

(b) $T$ commutes with shifts: $T_{n}\left(\Sigma^{j} M\right)=T_{n-j}(M)$ for each $n \in \mathbb{Z}$.

(c) $T\left(\mu_{s}^{M}\right)=\mu_{s}^{T(M)}$ for each $s \in S$, where $\mu_{s}$ denotes multiplication by $s$.

For the maximal ideal $\mathfrak{n}$ of $S$ property (c) implies:

2.4.1. One has $\mathfrak{n} \cdot T(k)=0$, so $T(k)$ is naturally a graded $k$-vector space.

Lemma 2.4.2. Let $\varphi: R \rightarrow S$ be a surjective homomorphism and $\epsilon: S \rightarrow k$ the canonical surjection. If $M$ is a complex of $S$-modules as in Theorem (2.3), then

$$
\nu_{S}\left(T_{n+i}(M)\right) \geq m \cdot \operatorname{rank}_{k} \operatorname{Im}\left(T_{n}(\epsilon)\right)
$$

Proof. First we simplify $M$. The inclusion into $M$ of the subcomplex

$$
M^{\prime}:=\quad \cdots \longrightarrow M_{i+2} \stackrel{\partial_{i+2}}{\longrightarrow} M_{i+1} \longrightarrow \operatorname{Ker}\left(\partial_{i}\right) \longrightarrow 0
$$

is a quasiisomorphism. By (a) one has $T_{n}(M) \cong T_{n}\left(M^{\prime}\right)$, so we may assume $M=M^{\prime}$. Set $H=\mathrm{H}_{i}(M)$, choose a surjection $H \rightarrow k^{m}$ and let $\pi$ denote the composition $M \rightarrow \Sigma^{i} H \rightarrow \Sigma^{i} k^{m}$ of morphisms of complexes of $S$-modules. Lifting $\Sigma^{i} \epsilon^{m}$ over $\pi$ to a morphism $\rho: \Sigma^{i} S^{m} \rightarrow M$, we get a commutative diagram

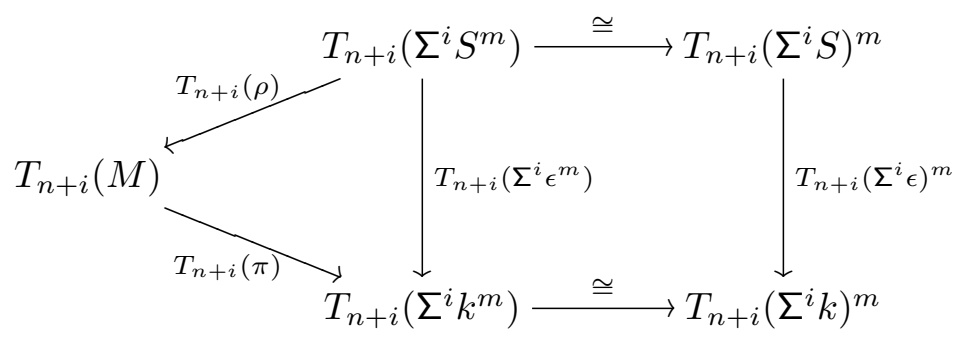


of homomorphism of $S$-modules. We can now write the relations below

$$
\begin{aligned}
\nu_{S}\left(T_{n+i}(M)\right) & \geq \operatorname{rank}_{k} \operatorname{Im}\left(T_{n+i}(\pi)\right) \\
& \geq \operatorname{rank}_{k} \operatorname{Im}\left(T_{n+i}\left(\Sigma^{i} \epsilon^{m}\right)\right) \\
& =m \cdot \operatorname{rank}_{k} \operatorname{Im}\left(T_{n+i}\left(\Sigma^{i} \epsilon\right)\right) \\
& =m \cdot \operatorname{rank}_{k} \operatorname{Im}\left(T_{n}(\epsilon)\right)
\end{aligned}
$$

by using consecutively the following facts: the maximal ideal of $S$ annihilates $T_{n+i}\left(\Sigma^{i} k\right)$; the diagram commutes; $T_{n+i}\left(\Sigma^{i} k\right)$ is isomorphic to $T_{n}(k)$.

We need an explicit description of a subcomplex of an acyclic closure $G$ of $\varphi$.

2.5. In the notation of (1.1), each $R$-module $G_{n}^{(2)}$ has a basis

$$
\left\{x_{\boldsymbol{i}} y_{\boldsymbol{j}} \mid \boldsymbol{i} \subseteq[1, c], \boldsymbol{j}=\left(j_{1}, \ldots, j_{d}\right) \in \mathbb{N}^{d}, \operatorname{card} \boldsymbol{i}+2 \sum_{h=1}^{d} j_{h}=n\right\}
$$

where $[1, c]=\{1, \ldots, c\}$. Let $\left\{a_{1}, \ldots, a_{c}\right\}$ be a minimal set of generators of $\operatorname{Ker} \varphi$ and $E$ the Koszul complex on it. The differential of $G^{(2)}$ then has the form

$$
\partial\left(x_{\boldsymbol{i}} y_{\boldsymbol{j}}\right)=\sum_{i \in \boldsymbol{i}} \pm a_{i} x_{\boldsymbol{i} \backslash\{i\}} y_{\boldsymbol{j}}+\sum_{i \in[1, c]} \sum_{j=1}^{d} \pm b_{i j} x_{\boldsymbol{i} \cup\{i\}} y_{\boldsymbol{j}-\boldsymbol{e}_{j}}
$$

where $\boldsymbol{e}_{j} \in \mathbb{N}^{d}$ is the $j$ th unit vector, and

$$
z_{j}=\sum_{i=1}^{c} b_{i j} x_{i} \in G_{1}^{(2)} \quad \text { for } \quad j=1, \ldots, c
$$

are cycles whose homology classes minimally generate $\mathrm{H}_{1}(E)$.

All the coefficients $a_{i}$ and $b_{i j}$ are in $\mathfrak{m}$ : this is clear for the $a_{i}$; as they minally generate the relation $0=\partial\left(z_{j}\right)=\sum_{i=1}^{c} b_{i j} a_{i}$ implies $b_{i j} \in \mathfrak{m}$.

Proof of Theorem (2.3). Let $\epsilon: S \rightarrow k$ be the canonical surjection. Lemma (2.4.2) applied to the functor $T$ defined by $T_{n}(M)=\operatorname{Tor}_{n}^{R}(S, M)$ yields

$$
\nu_{S}\left(\operatorname{Tor}_{n+i}^{R}(S, M)\right) \geq m \cdot \operatorname{rank}_{k} \operatorname{Im}\left(\operatorname{Tor}_{n}^{R}(S, \epsilon)\right)
$$

Next we estimate the rank on the right hand side. Let $G^{(2)}$ be the second stage in an acyclic closure of $\varphi, F$ a free resolution of $S$ over $R$, and let $\gamma: G^{(2)} \rightarrow F$ be a comparison morphism. The following diagram commmutes:

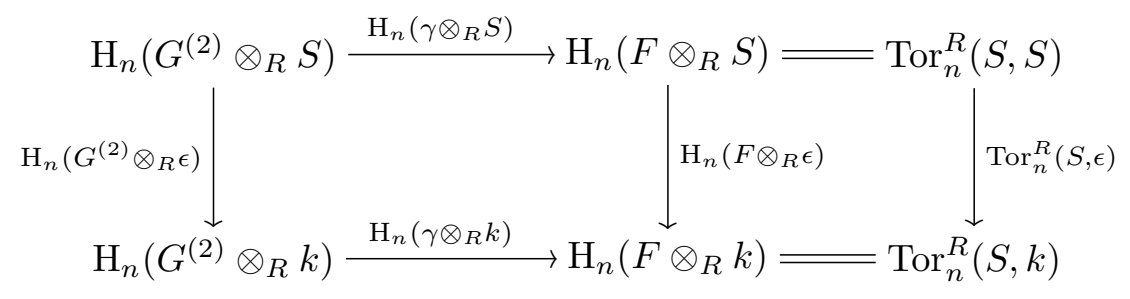


As $\mathrm{H}_{n}\left(\gamma \otimes_{R} k\right)$ is injective by (1.4.1), for each $n$ we get

$$
\operatorname{rank}_{k}\left(\operatorname{Im} \operatorname{Tor}_{n}^{R}(S, \epsilon)\right) \geq \operatorname{rank}_{k} \operatorname{Im}\left(\mathrm{H}_{n}\left(G^{(2)} \otimes_{R} \epsilon\right)\right)
$$

From the description of $G^{(2)}$ in (2.5) one sees that the graded submodule

$$
Z=\bigoplus_{\boldsymbol{i} \subseteq[1, c]} S\left(x_{\boldsymbol{i}} y_{0} \otimes 1\right) \oplus \bigoplus_{\boldsymbol{j} \in \mathbb{N}^{d} \backslash 0} S\left(x_{[1, c]} y_{\boldsymbol{j}} \otimes 1\right) \subseteq G^{(2)} \otimes_{R} S
$$

consists of cycles and the differential of $G^{(2)} \otimes_{R} k$ is trivial; thus the composition

$$
Z \otimes_{S} k \longrightarrow k \otimes_{S} \mathrm{H}\left(G^{(2)} \otimes_{R} S\right) \longrightarrow \mathrm{H}\left(G^{(2)} \otimes_{R} k\right)=G^{(2)} \otimes_{R} k
$$

is injective. Counting ranks over $k$ one obtains inequalities

$$
\begin{gathered}
\operatorname{rank}_{k} \operatorname{Im}\left(\mathrm{H}_{n}\left(G^{(2)} \otimes_{R} \epsilon\right)\right) \geq\left(\begin{array}{l}
c \\
n
\end{array}\right) \quad \text { for } \quad 0 \leq n \leq c \\
\operatorname{rank}_{k} \operatorname{Im}\left(\mathrm{H}_{2 n+c}\left(G^{(2)} \otimes_{R} \epsilon\right)\right) \geq\left(\begin{array}{c}
n+d-1 \\
n
\end{array}\right)
\end{gathered}
$$

To get the desired result, concatenate the (in)equalities established above.

Proof of Theorem (2.1). By hypothesis, one has $\operatorname{Tor}_{t}^{R}(S, M)=0=\operatorname{Tor}_{u}^{R}(S, M)$ for integers $t, u$ satisfying $t, u \geq \inf (\mathrm{H}(M))=i>-\infty$ and $t \not \equiv u(\bmod 2)$. The first inequality established in Theorem (2.3) implies $t, u>i+c$ for $c=\varepsilon_{2}(\varphi)$. For $d=\varepsilon_{3}(\varphi)$ the second inequality in the theorem then yields $\left(\begin{array}{c}n+d-1 \\ d-1\end{array}\right)=0$ for some $n \geq 1$, forcing $d=0$. Thus, $\varphi$ is complete intersection by (1.2.3).

\section{Vanishing of cohomology}

In this section we provide a cohomological criterion for a 2-closed homomorphism to be c.i. It uses a notion of depth of a complex $M$, defined by

$$
\operatorname{depth}_{S} M=\inf \left\{n \in \mathbb{Z} \mid \operatorname{Ext}_{S}^{n}(k, M) \neq 0\right\}
$$

This is the classical concept when $M$ is a finite $S$-module.

Theorem 3.1. Let $\varphi: R \rightarrow S$ be a 2-closed homomorphism and $M$ a complex of $S$-modules with $\mathrm{H}(M)$ degreewise finite and bounded above.

If there exist integers $t, u \geq \operatorname{depth}_{S} M-\operatorname{dim} S$, of different parity, such that $\operatorname{Ext}_{R}^{t+n}(S, M)=0=\operatorname{Ext}_{R}^{u+n}(S, M) \quad$ for $\quad 0 \leq n \leq \max \left\{\operatorname{dim}_{S} \mathrm{H}_{n}(M) \mid n \in \mathbb{Z}\right\}$ then the homomorphism $\varphi$ is complete intersection.

Remark. As one always has $\operatorname{dim} S-\operatorname{depth}_{S} M \geq \sup \mathrm{H}(M)$, see [12, (2.11.3)], the bound on $t, u$ in the theorem may be replaced by $t, u \geq-\sup \mathrm{H}(M)$.

Theorem (3.1) is a cohomological counterpart to Theorem (2.1), which provides a main ingredient in its proof. Another component is the use of properties of dualizing complexes, reviewed below; we refer to Hartshorne [15] for details. 
3.2. Dualizing complexes. A dualizing complex for $(S, \mathfrak{n}, k)$ is a complex

$$
D=0 \rightarrow D_{0} \rightarrow D_{-1} \rightarrow \cdots \rightarrow D_{-\operatorname{dim} S} \rightarrow 0
$$

of injective modules with $\mathrm{H}(D)$ degreewise finite and $\operatorname{Hom}_{S}(k, D) \simeq \Sigma^{-\operatorname{dim} S} k$.

Up to a quasiisomorphism of complexes, $S$ has at most one dualizing complex. Such a complex exists when the local ring $S$ is complete.

For each complex of $S$-modules $M$ we set $M^{\dagger}=\operatorname{Hom}_{S}(M, D)$.

3.2.1. If $\mathrm{H}(M)$ is degreewise finite, then so is $\mathrm{H}\left(M^{\dagger}\right)$.

3.2.2. If $\mathrm{H}(M)$ is bounded on one side, then $\mathrm{H}\left(M^{\dagger}\right)$ is bounded on the other.

Lemma 3.2.3. If $\mathrm{H}(M)$ is degreewise finite and bounded above, then

$$
\inf \mathrm{H}\left(M^{\dagger}\right)=\operatorname{depth}_{S} M-\operatorname{dim} S
$$

Proof. The complex $\mathrm{H}\left(M^{\dagger}\right)$ is degreewise finite and bounded below, see (3.2). This implies the first equality below; the second one holds by definition:

$$
\inf \mathrm{H}\left(M^{\dagger}\right)=\inf \mathrm{H}\left(k \otimes_{S}^{\mathbf{L}} M^{\dagger}\right)=\inf \mathrm{H}\left(k \otimes_{S}^{\mathbf{L}} \operatorname{Hom}_{S}(M, D)\right)
$$

To compute the right hand side we use a sequence of quasiisomorphisms:

$$
\begin{aligned}
k \otimes_{S}^{\mathbf{L}} \operatorname{Hom}_{S}(M, D) & \simeq \operatorname{Hom}_{S}\left(\operatorname{RHom}_{S}(k, M), D\right) \\
& \simeq \operatorname{Hom}_{S}\left(\operatorname{Ext}_{S}(k, M), D\right) \\
& \simeq \operatorname{Hom}_{k}\left(\operatorname{Ext}_{S}(k, M), \operatorname{Hom}_{S}(k, D)\right) \\
& \simeq \operatorname{Hom}_{k}\left(\operatorname{Ext}_{S}(k, M), \Sigma^{-\operatorname{dim} S} k\right) \\
& \simeq \Sigma^{-\operatorname{dim} S} \operatorname{Hom}_{k}\left(\operatorname{Ext}_{S}(k, M), k\right)
\end{aligned}
$$

The first one holds because $k$ has a resolution by finite free $S$-modules and $D$ is a bounded complex of injectives. For the second, note that $\mathbf{R H o m}_{S}(k, M)$ can be represented by a complex of $S$-modules annihilated by $\mathfrak{n}$, so it is quasiisomorphic to its own homology, namely, $\operatorname{Ext}_{S}(k, M)$. The third one holds because $\operatorname{Ext}_{S}(k, M)$ is a direct sum of copies of shifts of $k$. The fourth quasiisomorphism is induced by $\operatorname{Hom}_{S}(k, D) \simeq \Sigma^{-\operatorname{dim} S} k$; see (3.2). The last one is standard.

We now finish the computation of $\inf \mathrm{H}\left(M^{\dagger}\right)$ as follows:

$$
\begin{aligned}
\inf \mathrm{H}\left(M^{\dagger}\right) & =\inf \left(\Sigma^{-\operatorname{dim} S} \operatorname{Hom}_{k}\left(\operatorname{Ext}_{S}(k, M), k\right)\right) \\
& =\inf \operatorname{Hom}_{k}\left(\operatorname{Ext}_{S}(k, M), k\right)-\operatorname{dim} S \\
& =\operatorname{depth}_{S} M-\operatorname{dim} S
\end{aligned}
$$

3.2.4. For every finite $S$-module $N$ one has

$$
\operatorname{Ext}_{S}^{n}(N, D)=0, \quad \text { unless } \quad \operatorname{dim} S-\operatorname{dim}_{S} N \leq n \leq \operatorname{dim} S-\operatorname{depth}_{S} N
$$

3.3. The support of a complex $M$ is defined to be the set

$$
\operatorname{Supp}_{S} M=\left\{\mathfrak{q} \in \operatorname{Spec} S \mid \mathrm{H}\left(M_{\mathfrak{q}}\right)=0\right\}
$$

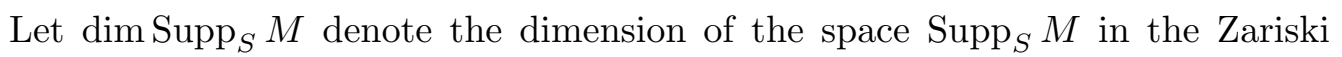
topology on Spec $S$. It is not hard to see that if $\mathrm{H}(M)$ is degreewise finite, then

$$
\operatorname{dim} \operatorname{Supp}_{S} M=\max \left\{\operatorname{dim}_{S} \mathrm{H}_{n}(M) \mid n \in \mathbb{Z}\right\}
$$


Proof of Theorem (3.1). By (1.4.3), the map $\widehat{\varphi}: \widehat{R} \rightarrow \widehat{S}$ of maximal-ideal-adic completions induced by $\varphi$ is 2-closed. For $\widehat{M}=M \otimes_{S} \widehat{S}$ and each $n \in \mathbb{Z}$ one has

$$
\mathrm{H}_{n}(\widehat{M}) \cong \mathrm{H}_{n}(M) \otimes_{R} \widehat{R} \quad \text { and } \quad \operatorname{Ext}_{\widehat{R}}^{n}(\widehat{S}, \widehat{M}) \cong \operatorname{Ext}_{R}^{n}(S, M) \otimes_{R} \widehat{R}
$$

where the first isomorphism is due to the flatness of $\widehat{R}$ over $R$, while the second uses, in addition, that $S$ is finite over $R$ and that $\mathrm{H}(M)$ is bounded above. In particular, one has $\operatorname{dim}_{\widehat{S}} \mathrm{H}_{n}(\widehat{M})=\operatorname{dim}_{S} \mathrm{H}_{n}(M)$ for each $n \in \mathbb{Z}$. Thus, the hypotheses of the theorem do not change when $R, S, M$ are replaced by $\widehat{R}, \widehat{S}$, $\widehat{M}$, respectively. Furthermore, if $\widehat{\varphi}$ is c.i., then so is $\varphi$. Thus, we may assume that the ring $S$ is complete, and hence that it has a dualizing complex $D$. Set $m=\max \left\{\operatorname{dim}_{S} \mathrm{H}_{n}(M) \mid n \in \mathbb{Z}\right\}$.

As $D$ is a bounded complex of injectives, there is a natural quasiisomorphism

$$
\operatorname{Hom}_{S}\left(\mathbf{R H o m}_{R}(S, M), D\right) \simeq S \otimes_{R}^{\mathbf{L}} \operatorname{Hom}_{S}(M, D)
$$

The composition of the functors on the left gives rise to a spectral sequence with

$$
{ }_{2} \mathrm{E}^{p, q}=\operatorname{Ext}_{S}^{-p}\left(\operatorname{Ext}_{R}^{q}(S, M), D\right) \quad \text { and } \quad{ }_{r} \mathrm{~d}^{p, q}:{ }_{r} \mathrm{E}^{p, q} \longrightarrow{ }_{r} \mathrm{E}^{p-r, q+r-1}
$$

As the $R$-module $S$ is finite, one has $\operatorname{Ext}_{R}^{q}(S, M)_{\mathfrak{q}} \cong \operatorname{Ext}_{R}^{q}\left(S, M_{\mathfrak{q}}\right)$ for each $\mathfrak{q} \in \operatorname{Spec} S$, so $\operatorname{Supp}_{S} \operatorname{Ext}_{R}^{q}(S, M) \subseteq \operatorname{Supp}_{S} M$, for each $q \in \mathbb{Z}$. Thus, one gets

$$
\operatorname{dim}_{S_{\mathfrak{q}}}\left(\operatorname{Ext}_{R}^{q}(S, M)_{\mathfrak{q}}\right) \leq \operatorname{dim} \operatorname{Supp}_{S} M=m
$$

where the equality comes from (3.3). Now (3.2.4) yields

$$
{ }_{2} \mathrm{E}^{p, q}=0 \quad \text { for } \quad p \notin[-\operatorname{dim} S,-\operatorname{dim} S+m]
$$

so the sequence converges. Formula $(*)$ shows that its abutment is equal to

$$
\mathrm{H}\left(S \otimes_{R}^{\mathbf{L}} \operatorname{Hom}_{S}(M, D)\right)=\operatorname{Tor}^{R}\left(S, M^{\dagger}\right)
$$

On the other hand, our hypothesis entails ${ }_{2} \mathrm{E}^{p, q}=0$ for

$$
t \leq q \leq t+m \quad \text { and } \quad u \leq q \leq u+m
$$

As a consequence, one obtains equalities

$$
{ }_{2} \mathrm{E}^{p, q}=0 \quad \text { whenever } \quad p+q=t \quad \text { or } \quad p+q=u
$$

They imply ${ }_{\infty} \mathrm{E}^{p, q}=0$ if $p+q=t$ or $p+q=u$, so convergence yields

$$
\operatorname{Tor}_{t}^{R}\left(S, M^{\dagger}\right)=0=\operatorname{Tor}_{u}^{R}\left(S, M^{\dagger}\right)
$$

In view of Lemma (3.2.3) and our hypothesis, the complex $M^{\dagger}$ satisfies

$$
\inf \mathrm{H}\left(M^{\dagger}\right)=\operatorname{depth}_{S} M^{\dagger}-\operatorname{dim} S \leq \min \{t, u\}
$$

Now Theorem (2.1), applied to $M^{\dagger}$, shows that $\varphi$ is complete intersection. 


\section{4. (Co)homology of algebra retracts}

Let $\varphi: R \rightarrow S$ be a homomorphism of noetherian rings.

A section of $\varphi$ is a homomorphism of rings $\psi: S \rightarrow R$ such that $\psi \circ \varphi=\mathrm{id}^{S}$; when such a homomorphism exits $S$ is said to be an algebra retract of $R$. Another way to describe this situation is to say that $R$ is a supplemented algebra over $S$. The study of homological and cohomological properties of supplemented algebras is a central topic in the classical literature on homological algebra.

Each $\mathfrak{q} \in$ Spec $S$ defines a local homomorphism $\varphi_{\mathfrak{q}}: R_{\varphi^{-1}(\mathfrak{q})} \rightarrow S_{\mathfrak{q}}$. If $\psi$ is a section of $\varphi$, the local homomorphism $\psi_{\mathfrak{p}}$, where $\mathfrak{p}=\varphi^{-1}(\mathfrak{q})$, is a section of $\varphi_{\mathfrak{q}}$. In particular, the homomorphism $\varphi_{\mathfrak{q}}$ is 2-closed; see (1.3.3).

Next we establish global versions of results from the preceding sections. To this end we recall the construction of certain canonical homomorphisms.

4.1. With $I=\operatorname{Ker}(\varphi)$, one has a canonical isomorphism of $S$-modules

$$
I / I^{2} \cong \operatorname{Tor}_{1}^{R}(S, S)
$$

The graded $S$-module $\operatorname{Tor}_{*}^{R}(S, S)$ has a natural structure of a strictly commutative graded $S$-algebra, see $[25,(2.7 .8)]$, so there is a homomorphism of graded $S$-algebras: $\lambda^{S}: \wedge_{S}\left(I / I^{2}\right) \rightarrow \operatorname{Tor}^{R}(S, S)$. Define $\lambda^{M}$ to be the composition

$$
\wedge_{S}\left(I / I^{2}\right) \otimes_{S} \mathrm{H}(M) \stackrel{\lambda^{S} \otimes_{S} \mathrm{H}(M)}{\longrightarrow} \operatorname{Tor}^{R}(S, S) \otimes_{S} \mathrm{H}(M) \longrightarrow \operatorname{Tor}^{R}(S, M)
$$

where the second arrow is a Künneth map. Let $\lambda_{M}$ denote the composition

$$
\operatorname{Ext}_{R}(S, M) \longrightarrow \operatorname{Hom}_{S}\left(\operatorname{Tor}^{R}(S, S), \mathrm{H}(M)\right) \longrightarrow \operatorname{Hom}_{S}\left(\wedge_{S}\left(I / I^{2}\right), \mathrm{H}(M)\right)
$$

where the first arrow is a Künneth map, and the second is $\operatorname{Hom}_{S}\left(\lambda^{S}, \mathrm{H}(M)\right)$

Theorem 4.2. Let $\varphi: R \rightarrow S$ be a homomorphism of rings that admits a section, and let $M$ be a complex of $S$-modules with $\mathrm{H}(M)$ finite. Set $I=\operatorname{Ker}(\varphi)$.

For each prime ideal $\mathfrak{q} \in \operatorname{Supp}_{S} M$ the following conditions are equivalent.

(i) The homomorphism $\varphi_{\mathfrak{q}}$ is complete intersection.

(ii $\left.{ }_{*}\right)$ The map $\left(\lambda^{M}\right)_{\mathfrak{q}}$ is bijective and the $S_{\mathfrak{q}}$-module $\left(I / I^{2}\right)_{\mathfrak{q}}$ is projective.

(iii $\left.{ }_{*}\right)$ For integers $t, u \geq \inf \mathrm{H}(M)_{\mathfrak{q}}$ of different parity one has

$$
\operatorname{Tor}_{t}^{R}(S, M)_{\mathfrak{q}}=0=\operatorname{Tor}_{u}^{R}(S, M)_{\mathfrak{q}}
$$

(ii*) The map $\left(\lambda_{M}\right)_{\mathfrak{q}}$ is bijective.

(iii* $^{*}$ For integers $t, u \geq \operatorname{depth}_{S_{\mathfrak{q}}} M_{\mathfrak{q}}-\operatorname{dim} S_{\mathfrak{q}}$ of different parity one has

$$
\operatorname{Ext}_{R}^{t+i}(S, M)_{\mathfrak{q}}=0=\operatorname{Ext}_{R}^{u+i}(S, M)_{\mathfrak{q}} \quad \text { for } \quad i=0, \ldots, \operatorname{dim} \operatorname{Supp}_{S_{\mathfrak{q}}} M_{\mathfrak{q}}
$$

Proof. Set $\mathfrak{p}=\varphi^{-1}(\mathfrak{q})$. The $R$-module $S$ is finite, so for each $n \in \mathbb{Z}$ one has

$$
\operatorname{Tor}_{n}^{R}(S, M)_{\mathfrak{q}} \cong \operatorname{Tor}_{n}^{R_{\mathfrak{p}}}\left(S_{\mathfrak{q}}, M_{\mathfrak{q}}\right) \quad \text { and } \quad \operatorname{Ext}_{R}^{n}(S, M)_{\mathfrak{q}} \cong \operatorname{Ext}_{R_{\mathfrak{p}}}^{n}\left(S_{\mathfrak{q}}, M_{\mathfrak{q}}\right)
$$

Therefore, each of the conditions listed above is local; moreover, any section of $\varphi$ localizes to a section of $\varphi_{\mathfrak{q}}$. Thus, changing notation we may assume that $\varphi$ is a local homomorphism and that $\mathfrak{q}$ is the maximal ideal of $S$. 
(i) $\Longrightarrow\left(\mathrm{ii}_{*}\right)$ and (ii*). Let $E$ be the Koszul complex on a minimal generating set of $I$. It satisfies $\partial(E) \subseteq I E$ and is a free resolution of $S$, as $\varphi$ is c.i. We get

$$
\operatorname{Tor}^{R}(S, S) \cong \mathrm{H}\left(E \otimes_{R} S\right)=E \otimes_{R} S
$$

so the $S$-module $\operatorname{Tor}_{1}^{R}(S, S)$ is free and $\lambda^{S}$ is bijective. Thus, $\operatorname{Tor}_{n}^{R}(S, S)$ is finite free and vanishes for $n<0$ or $n>\nu_{S}(I)$, so the Künneth homomorphisms

$$
\begin{gathered}
\operatorname{Tor}^{R}(S, S) \otimes_{S} \mathrm{H}(M) \longrightarrow \operatorname{Tor}^{R}(S, M) \\
\operatorname{Ext}_{R}(S, M) \longrightarrow \operatorname{Hom}_{S}\left(\operatorname{Tor}^{R}(S, S), \mathrm{H}(M)\right)
\end{gathered}
$$

are bijective. The definitions of $\lambda^{M}$ and $\lambda_{M}$ show that they are bijective as well.

$\left(\mathrm{ii}_{*}\right) \Longrightarrow\left(\mathrm{iii}_{*}\right)$, and $\left(\mathrm{ii}^{*}\right) \Longrightarrow\left(\mathrm{iii}^{*}\right)$. These implications are clear because the $S$-module $I / I^{2}$ is finite and $\mathrm{H}(M)$ is bounded.

$\left(\right.$ iii $\left._{*}\right)$ or $\left(\right.$ iii $\left.^{*}\right) \Longrightarrow(\mathrm{i})$. As $\varphi$ is closed by (1.3.3), Theorem (2.1), respectively, Theorem (3.1), shows that condition ( $\left.\mathrm{iii}_{*}\right)$, respectively, (iii*), implies $\varphi$ is c.i.

\section{Hochschild (co)homology}

Finally, we return to the subject in the title of this article. First we recall a classical interpretation of the functors in question; see e.g. [25, (9.1.5)].

5.1. Let $\eta: K \rightarrow S$ be a homomorphism of rings and let $\varphi^{S}: S \otimes_{K} S \rightarrow S$ be the homomorphism of rings given by $\varphi^{S}\left(s \otimes_{K} s^{\prime}\right)=s s^{\prime}$.

If the $K$-module $S$ is flat, then for each $n \in \mathbb{Z}$ one has

$$
\operatorname{HH}_{n}(S \mid K ; M)=\operatorname{Tor}_{n}^{S \otimes_{K} S}(S, M)
$$

Note that $I / I^{2}$, where $I=\operatorname{Ker}\left(\varphi^{S}\right)$, is a standard realization of the module of differentials $\Omega_{S \mid K}$. The map $\lambda^{M}$ from (4.1) yields $S$-linear maps

$$
\lambda_{n}^{M}: \wedge_{S}^{n} \Omega_{S \mid K} \otimes_{S} M \longrightarrow \operatorname{HH}_{n}(S \mid K ; M)
$$

If $S$ is projective as a $K$-module, then also

$$
\operatorname{HH}^{n}(S \mid K ; M)=\operatorname{Ext}_{S \otimes_{K} S}^{n}(S, M)
$$

so in this context the homomorphism $\lambda_{M}$ from (4.1) reads

$$
\lambda_{M}^{n}: \operatorname{HH}^{n}(S \mid K ; M) \longrightarrow \operatorname{Hom}_{S}\left(\wedge_{S}^{n} \Omega_{S \mid K}, M\right)
$$

The maps above are the homomorphisms that appear in the introduction. For the proof of the theorem stated there we need a characterization of smoothness proved by André [1, Proposition C], using André-Quillen homology. A short version of his argument may be found in $[4,(1.1)]$.

5.2. A flat algebra $S$ essentially of finite type over a noetherian ring $K$ is smooth if and only if the homomorphism $\left(\varphi^{S}\right)_{\mathfrak{p}}$ is c.i., for each $\mathfrak{p} \in \operatorname{Spec} S$. 
Proof of the Main Theorem. Let $\varphi^{S}: S \otimes_{K} S \rightarrow S$ be the product map. We claim that, for a given $\mathfrak{q} \in \operatorname{Spec} S$, condition (i): the $K$-algebra $S_{\mathfrak{q}}$ is smooth, is equivalent to: (i') the homomorphism $\left(\varphi^{S}\right)_{\mathfrak{q}}:\left(S \otimes_{K} S\right)_{\left(\varphi^{S}\right)^{-1}(\mathfrak{q})} \rightarrow S_{\mathfrak{q}}$ is c.i.

Indeed, $\left(\varphi^{S}\right)_{\mathfrak{q}}$ is surjective, so it is c.i. if and only if $\left(\varphi^{S}\right)_{\mathfrak{p}}$ is c.i. for each $\mathfrak{p} \subseteq \mathfrak{q}$. However, the local homomorphisms $\left(\varphi^{S}\right)_{\mathfrak{p}}$ and $\left(\varphi^{S_{\mathfrak{q}}}\right)_{\mathfrak{p}}$ coincide, and the latter is c.i. for each $\mathfrak{p}$ precisely when the $K$-algebra $S_{\mathfrak{q}}$ is smooth, by (5.2).

Given this translation and the identifications in (5.1), the desired result is contained in Theorem (4.2), for $s \mapsto 1 \otimes s$ gives a section $S \rightarrow S \otimes_{K} S$ of $\varphi^{S}$.

The example below shows that condition $\left(\mathrm{ii}_{*}\right)$ in the Main Theorem cannot be weakened in general. We do not know whether the conclusion of the theorem still holds if the vanishing intervals in condition (iii*) are shortened.

Example 5.3. Let $S=\mathbb{Z}[\sqrt{2}]$. The Hochschild homology of $S$ over $\mathbb{Z}$ is

$$
\mathrm{HH}_{n}(S \mid \mathbb{Z} ; S)= \begin{cases}S & \text { for } n=0 \\ S /(2 \sqrt{2}) & \text { for odd } n \geq 1 \\ 0 & \text { for even } n \geq 2\end{cases}
$$

while the Hochschild cohomology of $S$ over $\mathbb{Z}$ is given by

$$
\mathrm{HH}^{n}(S \mid \mathbb{Z} ; S)= \begin{cases}S & \text { for } n=0 \\ 0 & \text { for odd } n \geq 1 \\ S /(2 \sqrt{2}) & \text { for even } n \geq 2\end{cases}
$$

Indeed, $\operatorname{Ker}\left(S \otimes_{\mathbb{Z}} S \rightarrow S\right)$ is generated by $\sqrt{2} \otimes 1-1 \otimes \sqrt{2}$. A free resolution of $S$ as a module over $S \otimes_{\mathbb{Z}} S$ is given by the complex $F$ below:

$\cdots \rightarrow S \otimes_{\mathbb{Z}} S \stackrel{\sqrt{2} \otimes 1-1 \otimes \sqrt{2}}{\longrightarrow} S \otimes_{\mathbb{Z}} S \stackrel{\sqrt{2} \otimes 1+1 \otimes \sqrt{2}}{\longrightarrow} S \otimes_{\mathbb{Z}} S \stackrel{\sqrt{2} \otimes 1-1 \otimes \sqrt{2}}{\longrightarrow} S \otimes_{\mathbb{Z}} S \rightarrow 0$

As $S$ is finite free as a $\mathbb{Z}$-module, $\operatorname{HH}_{*}(S \mid \mathbb{Z} ; S)$ is the homology of the complex

$$
F \otimes_{S \otimes_{\mathbb{Z}} S} S=\cdots \longrightarrow \stackrel{2 \sqrt{2}}{\longrightarrow} S \stackrel{0}{\rightarrow} S \stackrel{2 \sqrt{2}}{\longrightarrow} S \stackrel{0}{\rightarrow} S \longrightarrow 0
$$

and $\mathrm{HH}^{*}(S \mid \mathbb{Z} ; S)$ is the homology of the complex

$$
\operatorname{Hom}_{S \otimes_{\mathbb{Z}} S}(F, S)=0 \longrightarrow S \stackrel{0}{\rightarrow} S \stackrel{2 \sqrt{2}}{\longrightarrow} S \stackrel{0}{\rightarrow} S \stackrel{2 \sqrt{2}}{\longrightarrow} S \longrightarrow \cdots
$$

see (5.1). The desired expressions follow.

\section{References}

[1] M. André, Algèbres graduées associées et algèbres symétriques plates, Comment. Math. Helv. 49 (1974) 277-301.

[2] L. L. Avramov, Infinite free resolutions, Six lectures on commutative algebra (Bellaterra, 1996), 1-118, Progr. Math., 166, Birkhuser, Basel, 1998.

[3] L. L. Avramov, H.-B. Foxby, Cohen-Macaulay properties of ring homomorphisms, Adv. Math. 133 (1998), 54-95.

[4] L. L. Avramov, S. Iyengar, Homological criteria for regular homomorphisms and for locally complete intersection homomorphisms, Algebra, arithmetic and geometry, Part I, II (Mumbai, 2000), 97-122, Tata Inst. Fund. Res. Stud. Math., 16, Tata Inst. Fund. Res., Bombay, 2002. 
[5] L. L. Avramov, S. Iyengar, André-Quillen homology of algebra retracts, Ann. Sci. École Norm. Sup. (4) 36 (2003), no. 3, 431-462.

[6] L. L. Avramov, M. Vigué-Poirrier, Hochschild homology criteria for smoothness, Internat. Math. Res. Notices 2 (1992) 17-25.

[7] Buenos Aires Cyclic Homology Group, A Hochschild homology criterium for the smoothness of an algebra, Comment. Math. Helv. 69 (1994) 163-168.

[8] A. Blanco, J. Majadas, Sur l'annulation de la cohomologie de Hochschild des anneaux de Gorenstein, Comm. Algebra 24 (1996) 1777-1783.

[9] A. Blanco, J. Majadas, A. G. Rodicio, On the acyclicity of the Tate complex, J. Pure Appl. Algebra, 131 (1998) 125-132.

[10] W. Bruns, On the Koszul algebra of a local ring, Illinois J. Math. 37 (1993) 278-283.

[11] R.-O. Buchweitz, E. L. Green, D. Madsen, Ø. Solberg, Finite Hochschild cohomology without finite global dimension, Math. Res. Lett. 12 (2005), no. 5-6, 805-816.

[12] H.-B. Foxby, S. Iyengar, Depth and amplitude of unbounded complexes, Commutative algebra (Grenoble/Lyon, 2001), 119-137, Contemp. Math., 331, Amer. Math. Soc., Providence, RI, 2003.

[13] T. H. Gulliksen, A proof of the existence of minimal algebra resolutions, Acta Math. 120 (1968) 53-58.

[14] T. H. Gulliksen, G. Levin, Homology of local rings, Queen's Papers Pure Appl. Math. 20, Queen's Univ., Kingston, ON, 1969.

[15] R. Hartshorne, Residues and duality, Lecture Notes Math. 20, Springer-Verlag, BerlinNew York, 1966.

[16] D. Happel, Hochschild cohomology of finite-dimensional algebras, Séminaire d'Algèbre Paul Dubreil et Marie-Paul Malliavin (Paris, 1987/1988), Lecture Notes Math. 1404, Springer, Berlin, 1989; pp. 108-126.

[17] C. Huneke, J. Koh, Some dimension 3 cases of the Canonical Element Conjecture, Proc. Amer. Math. Soc. 98 (1986) 394-398.

[18] G. Hochschild, B. Kostant, A. Rosenberg, Differential forms on a regular affine algebra, Trans. Amer. Math. Soc. 102 (1962) 383-408.

[19] M. Hochster, Canonical elements in local cohomology modules and the direct summand conjecture, J. Algebra 84 (1983) 503-553.

[20] G. Levin, Large homomorphisms of local rings, Math. Scand. 46 (1980) 209-215.

[21] P. Roberts, The equivalence of two forms of the Canonical Element Conjecture, undated manuscript.

[22] A. G. Rodicio, Commutative augmented algebras with two vanishing homology modules, Adv. Math. 111 (1995) 162-165.

[23] C. Schoeller, Homologie des anneaux locaux noethériens, C. R. Acad. Sci. Paris Sér. A 265 (1967) 768-771.

[24] J. Tate, Homology of Noetherian rings and local rings, Illinois J. Math. 1 (1957) 14-25.

[25] C. Weibel, An introduction to homological algebra, Cambridge Stud. Adv. Math. 38, Cambridge Univ. Press, Cambridge, 1994.

Department of Mathematics, University of Nebraska, Lincoln, NE 68588

E-mail address: avramov@math.unl.edu, iyengar@math.unl.edu 